IRSTI 29.19.17; 29.19.95

\title{
Studies on gas release from pre-saturated samples on a plasma beam installation
}

\author{
T.R. Tulenbergenov ${ }^{1}$, T.V.Kulsartov ${ }^{1 *}$, \\ I.A. Sokolov ${ }^{1}$, B.K. Rakhadilov ${ }^{1}$, \\ D.A. Ganovichev ${ }^{1}$, A.J. Miniyazov ${ }^{1}$ and A.A. Sitnikov ${ }^{2}$ \\ ${ }^{1}$ Institute of Atomic Energy, RSE NNC RK, Kurchatov 071100, Kazakhstan \\ ${ }^{2}$ Polzunov Altai State Technical University, Barnaul 656000, Russian Federation \\ e-mail:lab243@mail.ru
}

\begin{abstract}
We conducted methodological studies to evaluate the possibility of conducting experiments on gas release from pre-saturated samples on a plasma-beam installation. One of the tasks of obtaining reliable data on the parameters of the interaction of hydrogen isotope ions with materials of the NNR is to conduct methodically correct post-test experiments on the study of gas release from the investigated samples subjected to ion irradiation. The paper presents three stages of research. In the first stage, experiments were con-ducted on saturation and gas release with the sample from tungsten. At the second stage, experiments were performed with a tungsten sample on saturation with its deuterium and degassing under stepwise heating conditions. At the third stage, experiments were conducted on gas release with vanadium samples, with maximum sensitivity for dissolved gas in the sample. The results of calculations of the temperature field on the sample are presented. It is shown that, on the whole, it reflects the experimentally observed effect on a significant sample temperature gradient.
\end{abstract}

Key words: tungsten, degassing, vanadium, plasma, temperature gradient, saturation, deuterium.

PACS numbers: 85.40.Ry; 82.56.Lz; 82.30.Rs; 88.85.mh; 28.52.-s.

\section{Introduction}

Main points which impact on received data quality were defined for different research methods of gas release from samples after ionic radiation. Brief list of them:

A) Sample pre saturation should be conducted under controlled conditions, i.e. should have adequate information on main radiation parameters:

- geometry of irradiation;

- ion flux parameter data (ion flux density, energy spectrum of ion flux);

- temperature field data in the volume of irradiation sample during radiation;

- data on irradiation time.

B) Procedures of post irradiated sample preparation on gas release should be optimal from the aspect of obtaining highest adequate information on gas release experiments. Following aspects are important according to numerous studies:

- time of sample aging after irradiation, before gas release experiments should be possibly minimal;
- irradiated sample contact with atmospheric gas was not desirable.

C) Gas release experiments of irradiated samples should be conducted under conditions permitting to define correctly gas flow from researched sample and depending on its temperature.

It is obviously, that for effective research on defining parameter of plasma impact on fusion reactor material sample it is desirable to have method conduction of gas release experiments directly after irradiation on the same installation on which irradiation was conducted.

\section{Material and methods}

\subsection{Experiments with tungsten samples}

Researches were conducted on plasma beam installation (scheme is in Figure 1) using target cooling device in which different researched samples were set.

Following tasks were solved during the experiment: 
1) determining of sample temperature at different places, for different heating modes of samples by electron and ion beam;

2) estimation of installation system operation impact on registration of mass-spectrometer data;

3 ) estimation of background gas release into an installation chamber for different heating modes of samples under continuous pumping conditions;

4) obtaining depending on gas release of dissolved hydrogen from vanadium sample under sample stage heating by electron beam;

5) receiving of gas release depending saturated from deuterium ion flux from vanadium sample under sample stage heating by electron beam.

Experiments with tungsten samples were primarily conducted, for which mode parameters of sample temperature heating dimensions of $10 \mathrm{~mm}$ diameter; $4.5 \mathrm{~mm}$ thickness were estimated; data on composition of gaseous phase in interaction chamber for different stages of operation set of plasma beam installation under maximal pumping of chamber mode were given; experiments on saturation of tungsten sample by deuterium were carried out and gas release experiment under stage heating conditions was conducted.

Tungsten sample was chosen due to its properties. It follows from the literature sources (see Figure 2) on dissolution of hydrogen isotopes in given material (equally with molybdenum and other refractory metals and melts), that given material has one of the lowest volume in dissolution constant (less than in stainless steel for more than 2 order of magnitude).

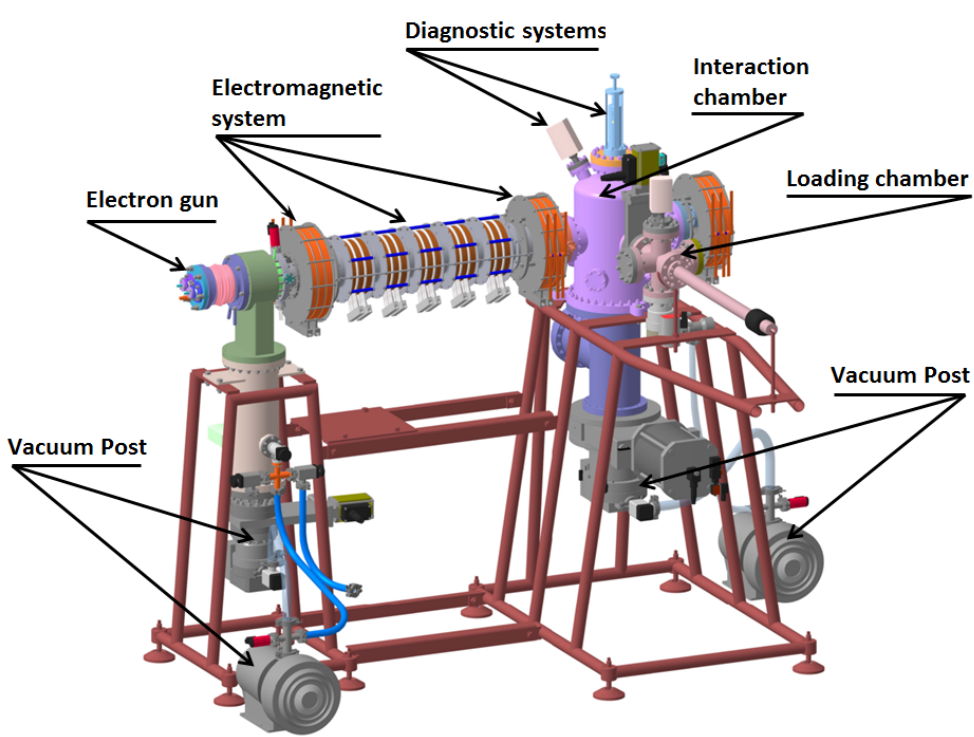

Figure1 - Structural layout of plasma beam installation of simulation bench

Therefore, saturation and gas release experiments with researched sample from tungsten modeled in great amount condition impact of irradiated experiment on hydrogen isotope and target materials interaction, but not samples, defining lower border of possible background impact on such interaction.

Tungsten sample with $10 \mathrm{~mm}$ diameter and 4.5 $\mathrm{mm}$ thickness was set into target unit by molybdenum holder. CA typed thermocouple was set on sample reverse side.

Initially depending on gas composition changing in interaction chamber on serial turning on of all installation systems were taken: from cooling supply in electron beam gun chamber; back heating in cathode-breaker assembly; tablets; electro-magnetic system turning on; and creating heat up sample of electron beam by accelerating voltage changing for different values of sample acceleration voltages and currents. Experiments were conducted on scanning electron beam with $3 \mathrm{~mm}$ diameter on sample center, and on electron beam defocusing up to $30 \mathrm{~mm}$ diameter.

Main received experiment parameters were given in Table 1 and Figures 3, 4. 


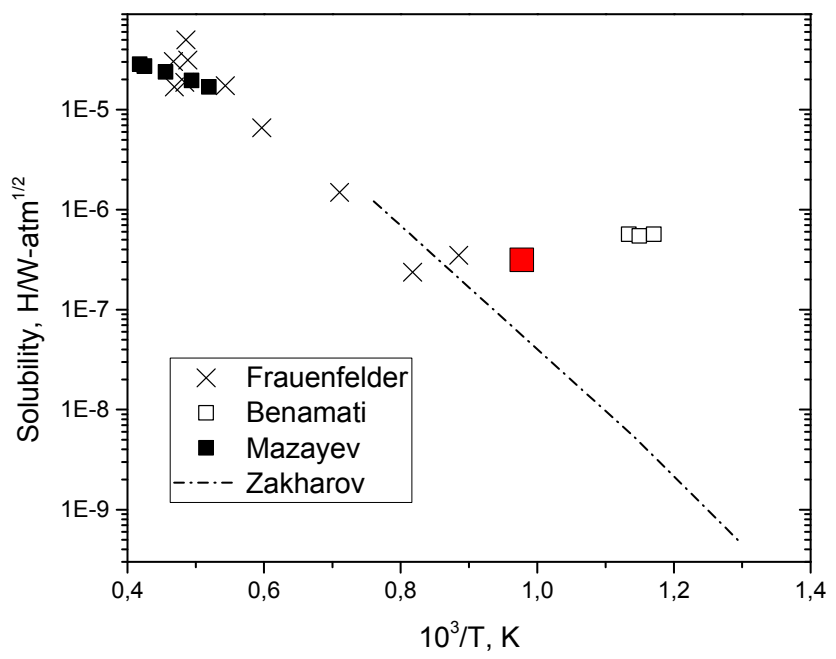

Figure 2 - Temperature depending of hydrogen solution in tungsten [4-7]

Table 1 - Experiment data on heating of tungsten sample

\begin{tabular}{|c|c|c|c|c|c|}
\hline $\begin{array}{c}\text { Temperature on } \\
\text { sample front side, } \mathrm{C}\end{array}$ & $\begin{array}{c}\text { Temperature on } \\
\text { sample reverse side, } \\
\text { C }\end{array}$ & $\begin{array}{c}\text { Pressure in chamber } \\
\text { with electron gun; } \\
* 10^{-6} \text {, Torr }\end{array}$ & $\begin{array}{c}\text { Pressure in } \\
\text { interaction } \\
\text { chamber } \\
* 10^{-6} \text {, Torr }\end{array}$ & $\begin{array}{c}\text { Accelerating } \\
\text { voltage, } \mathrm{V}\end{array}$ & $\begin{array}{c}\text { Current on sample, } \\
\mathrm{mA}\end{array}$ \\
\hline \multicolumn{6}{|c|}{ Electron beam diameter of $3 \mathrm{~mm}$} \\
\hline & 396 & 2.62 & 1.67 & 1000 & 12 \\
\hline 866 & 521 & 2.67 & 2.21 & 1200 & 15 \\
\hline 980 & 579 & 2.68 & 2.58 & 1400 & 16 \\
\hline 1026 & 596 & 2.71 & 2.11 & 1600 & 19 \\
\hline 992 & 583 & 2.67 & 1.61 & 1400 & 17 \\
\hline 848 & 510 & 2.64 & 1.23 & 1200 & 14 \\
\hline- & 414 & 2.65 & 1.07 & 1000 & 12 \\
\hline 975 & 576 & 2.63 & 1.3 & 1400 & 17 \\
\hline \multicolumn{6}{|c|}{ Electron beam diameter of $30 \mathrm{~mm}$} \\
\hline & 440 & 2.63 & 1.18 & 1400 & 17 \\
\hline
\end{tabular}

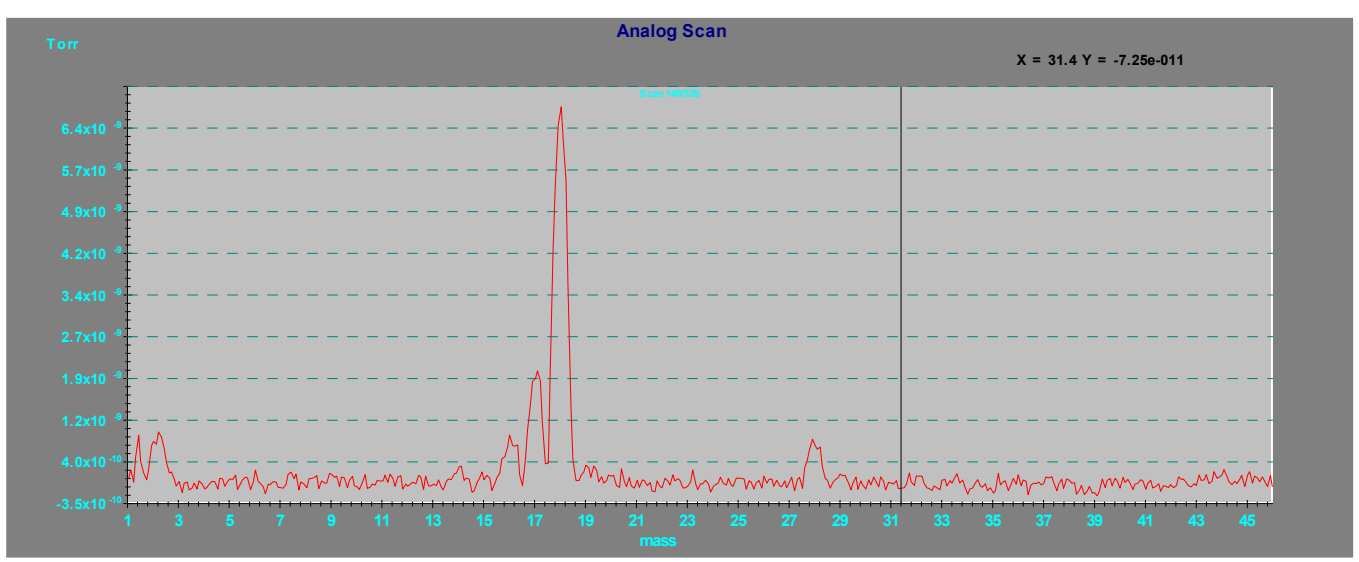

Figure 3 - Typical gas spectrum in interaction chamber under tungsten sample heating by electron beam 


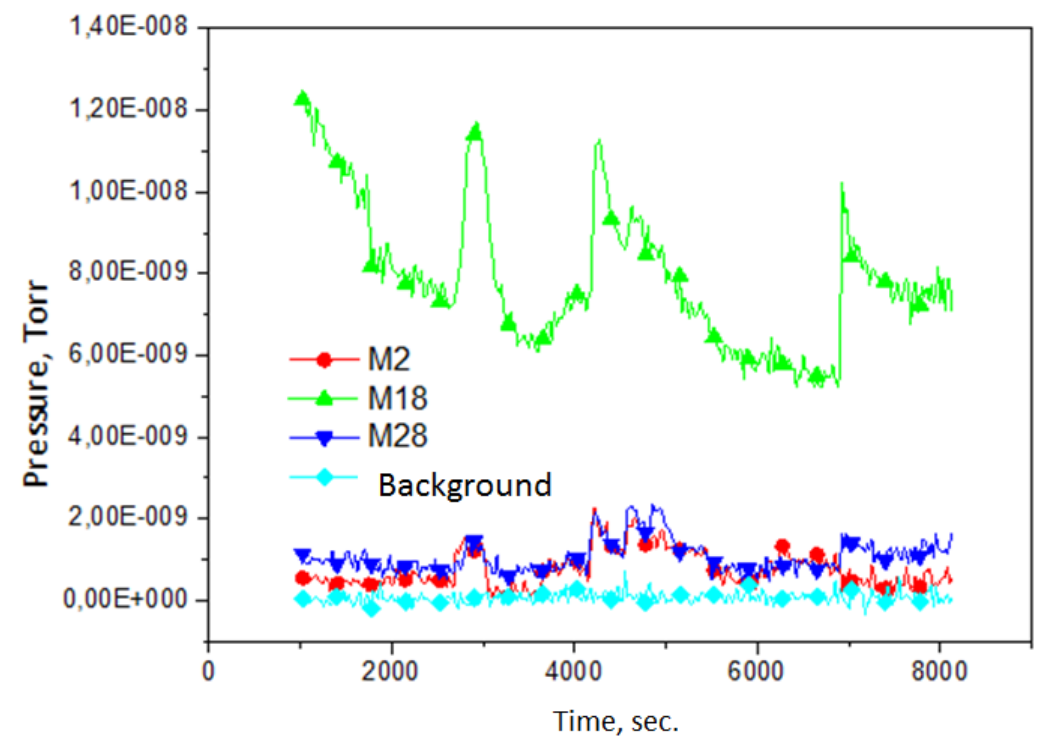

Figure 4 - Change depending of gaseous phase composition in interaction chamber under stage heating of tungsten sample by electron beam

Conclusions on experiment first stage with tungsten samples include the following:

1) Main peaks, observed for interaction chamber on mass-spectrometer are water (M18), nitrogen and carbon monoxide (CO) (M28) and hydrogen peaks;

2) Water peak (characterized for spectrum of residual gas of vacuum chamber, not exposed to burn-in) which is gradually decreased on chamber pumping, but is increased during target unit heating, by demonstrating peak release. Minor increase of hydrogen and carbon monoxide peaks are also observed under heating, which connects with gas release upon sample and target unit heating;

3) Nevertheless, general level of residual gas is not enough high (on hydrogen is less than $2 * 10^{-9}$ Torr for any heating mode) and permit to conduct gas release experiments;

4) different sample temperatures with accuracy of about $10{ }^{\circ} \mathrm{C}$ can be effectively set by accelerating voltage of electron beam heating sample;

5) there is marked temperature gradient through sample thickness of about $300{ }^{\circ} \mathrm{C}$ for front surface sample begin with $850^{\circ} \mathrm{C}$ and above;

6) Electron beam defocussing leads to significant decrease of sample temperature more than $200{ }^{\circ} \mathrm{C}$ for same values of accelerating voltages.

Experiments on tungsten sample saturation with deuterium and degasification under the stage heating conditions by electron beam were conducted on the second stage of the experiment.
Experiments on saturation above all should allow to estimate sample temperature level during its heating by ion and electron beam. The main experimental parameters obtained are given in Table 2.

Saturation was conducted under deuterium pressure in recharging chamber about $3-5 \cdot 10^{-3}$ Torr; $500 \mathrm{~V}$ potential was given to sample upon saturation. Average saturation time was about 5 minutes on every temperature shelves.

Directly after saturation, interaction chamber was pumped from deuterium and gas release experiment was conducted.

Sample was heated initially by $30 \mathrm{~mm}$ diameter electron beam, then by $8 \mathrm{~mm}$ diameter electron beam. Mass-spectrum changes were registered, in particular peak changes corresponding to $\mathrm{HD}$, deuterium hydrogen and water molecules. (see Figure 5).

Conclusions on the second stage of tungsten sample include the following:

1) deuterium release flow from tungsten sample could not fixed predictably after plasma impact on its subsequent heating; deuterium release from other target unit elements was also not fixed correspondingly, which allows to conclude that deuterium background level in chamber will not exceed $10^{-9}$ Torr for gas release experiments

2) different sample temperatures with accuracy of about $10{ }^{\circ} \mathrm{C}$ under its saturation from plasma discharge can be effectively set by accelerating voltage of electron beam heating sample 
3) there is marked temperature gradient through sample thickness under its saturation of plasma flow which consists $300^{\circ} \mathrm{C}$ for front surface sample begin with $850{ }^{\circ} \mathrm{C}$ and above.

Therefore, preliminary stage of methodic experiments was finished which leads to obtain approach to conduct method on gas release experiments on plasma beam installation, number of parameters relate to temperature fields on sample volume under its saturation and degasification conditions, electron beam parameters providing necessary temperature samples were received, background values of gas composition in chamber with sample during conducting gas release experiments were estimated for researching saturation/degasification mode.

Table 2 - Test data on tungsten sample saturation by deuterium

\begin{tabular}{|c|c|c|c|c|c|}
\hline $\begin{array}{c}\text { Temperature on } \\
\text { sample front side, } \\
\text { C }\end{array}$ & $\begin{array}{c}\text { Temperature on } \\
\text { sample reverse } \\
\text { side, C }\end{array}$ & $\begin{array}{c}\text { Pressure in } \\
\text { chamber with } \\
\text { electron gun; } * 10^{-} \\
\quad \text {, Torr }\end{array}$ & $\begin{array}{c}\text { Accelerating } \\
\text { voltage, } \mathrm{V}\end{array}$ & $\begin{array}{c}\text { Current on a } \\
\text { target, } \mathrm{MA}\end{array}$ & $\begin{array}{l}\text { Current on } \\
\text { sample, } \mathrm{mA}\end{array}$ \\
\hline \multicolumn{6}{|c|}{ Electron beam diameter of $30 \mathrm{~mm}$} \\
\hline & 141 & 2.5 & 1900 & 62 & 28 \\
\hline & 209 & 2.7 & 2200 & 78 & 32 \\
\hline \multicolumn{6}{|c|}{ Electron beam diameter of $8 \mathrm{~mm}$} \\
\hline & 312 & 2.63 & 1500 & 41 & 19 \\
\hline & 460 & 2.4 & 1900 & 59 & 26 \\
\hline 835 & 530 & 2.4 & 2000 & 74 & 28 \\
\hline 878 & 546 & 2.6 & 2200 & 78 & 30 \\
\hline
\end{tabular}

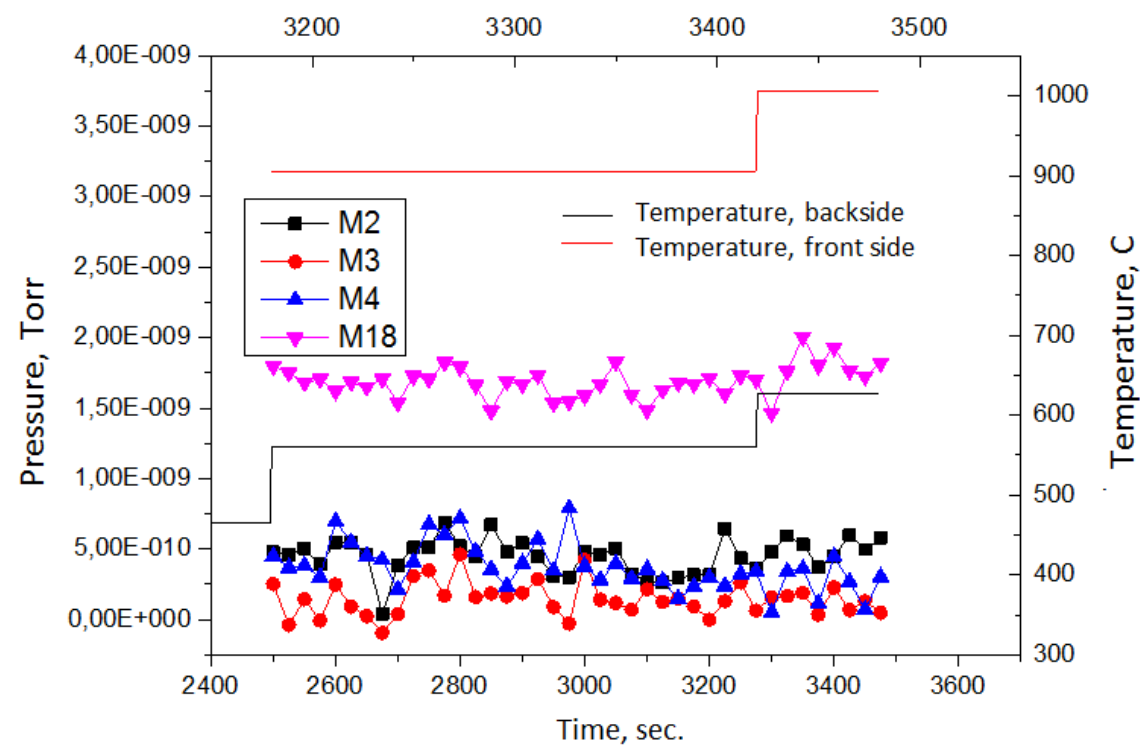

Figure 5 - Depending of gas phase composition change in interaction chamber under stage heating of tungsten sample after plasma impact

\subsection{Experiments with vanadium samples.}

Experiments with vanadium samples were conducted on the second stage: first sample was similar to tungsten sample (dimensions of $10 \mathrm{~mm}$ diameter, $4.5 \mathrm{~mm}$ thickness); second sample was thinner (dimensions of $10 \mathrm{~mm}$ diameter, $2 \mathrm{~mm}$ thickness). Purpose of experiments was to define experiment mode parameters on gas release of preliminary saturated samples with maximal sensitivity of dissolved gas in sample. 
Vanadium sample material was chosen for these purposes. Vanadium has high parameter values of hydrogen isotope dissolution (Table 3). Hydrogen dissolution in vanadium is more than 7 order of magnitude high, in comparison with tungsten. Being hydride-forming material, vanadium is capable to absorb water in significant amount - volume hydrogen concentration in vanadium hydride exceeds hydrogen concentration in liquefied condition. In addition, relatively small hydrogen concentration is presented in it. All this together makes it convenient material for saturation and gas release experiments.

Table 3 - Parameters of hydrogen isotopes dissolution in vanadium

\begin{tabular}{|c|c|c|c|}
\hline Source & Isotope & So $_{0}$ at. fraction $\left.\mathrm{Pa}^{1 / 2}\right)$ & Es $(\mathrm{meV})$ \\
\hline$[8] 120-470^{\circ} \mathrm{C}$ & $\mathrm{H}$ & $2.2 \mathrm{E}-6$ & -331 \\
\hline$[9] 220-550^{\circ} \mathrm{C}$ & $\mathrm{H}$ & $1.6 \mathrm{E}-6$ & -353 \\
\hline$[10] 600-1200^{\circ} \mathrm{C}$ & $\mathrm{H}$ & $3.6 \mathrm{E}-6$ & -293 \\
\hline$[11] 20-150^{\circ} \mathrm{C}$ & $\mathrm{H}$ & $1.7 \mathrm{E}-6$ & -344 \\
\hline$[12] 200-450^{\circ} \mathrm{C}$ & $\mathrm{H}$ & $2.1 \mathrm{E}-6$ & -330 \\
\hline$[13] 700-1200^{\circ} \mathrm{C}$ & $\mathrm{H}$ & $1.7 \mathrm{E}-6$ & -344 \\
\hline$[14] 30-700^{\circ} \mathrm{C}$ & $\mathrm{H}$ & $2.4 \mathrm{E}-6$ & -331 \\
& $\mathrm{D}$ & $1.9 \mathrm{E}-6$ & -341 \\
\hline
\end{tabular}

Experiments with first vanadium sample concluded on following: after sample installation water was pumped and sample was heated in stages by electron beam. Experiments were conducted under different beam parameters and different modes of interaction chamber pumping. Mode with one TMP pumping was mainly used which is set on massspectrometer for increasing system sensitivity of mass-spectrometer registration. Changes of gas composition in chamber were registered during experiments.

One of the first stage heating samples allows fixing significant release of dissolved hydrogen in vanadium sample (see Figure 6). Hydrogen release character completely corresponds to expect one: on first electron beam supply by accelerating voltage about $1000 \mathrm{~V}$ on sample it begins slowly heated and we observe pressure peak and growth of released hydrogen at initial time depending on sample warmup. Further on increasing of accelerating voltage up to $1400 \mathrm{~V}$, great peak of hydrogen release was observed which declines with time (temperature balance set is more faster than on the first warm-up as sample temperature is significantly higher); and on last accelerating voltage increase there was observed last hydrogen peak which is less than previous (as hydrogen basic amount is already released from sample probably). At the expense of diffusion speed increase with sample temperature hydrogen release decay for second peak is more "fast".
It should be noted there, that obtained experiment results on vanadium sample degasification, carried out on high sensitivity of registration system were completely approved principal possibility of gas release experiment conduction on plasma-beam installation.

Experiments with second vanadium samples were conducted for further task on defining optimal gas release method on plasma-beam installation. Second researched sample was thinner than the first, by reason of increasing method correctness:

1) for temperature and concentration gradient reduction on sample under its saturation in ion flux and warm-up by electron beam;

2) for reduction of temperature balance occurrence on sample upon its saturation in ion flux on electron beam warm-up;

3) for more correct processing of obtained gas release depending, here we have to clarify that mathematical apparatus of atom migration was well developed in present, ratio of average linear dimension (in this case diameter) to sample thickness, in which is no more than 4. In other cases, gas release depending processing should conducted by special design/model programs.

After second vanadium sample installation, its stage annealing was conducted under continuous pumping and mass-spectrometer registration conditions. Figure 7 shows depending change of hydrogen pressure in chamber during annealing. As 
can be seen from figure, hydrogen release depending similar to previous obtained one. It is seen, that at the annealing end, the sample was degasified from dissolved in it hydrogen.

Saturation of vanadium sample by deuterium was conducted after annealing. Saturation was conducted in 30 minutes under deuterium pressure in chamber of $5^{*} 10^{-3}$ Torr on sample temperature of about 160 ${ }^{\circ} \mathrm{C}$. Electron beam was on defocussing mode (diameter of $30 \mathrm{~mm}$ ); accelerating voltage of $1400 \mathrm{~V}$; beam current of $16 \mathrm{~mA}$; current on a target of $58 \mathrm{~mA}$. Potential of $1000 \mathrm{~V}$ was given to a target unit.

Further experiment on deuterium gas release from saturated vanadium sample was conducted. Experiment diagram is shown in Figure 8. It is seen that experiment allowed to obtain qualitative gas release kinetics of deuterium and HD molecules. It turned out that deuterium quantity entered in sample in the result of interaction with ion flux was about $3 * 10^{-6} \mathrm{~mol}$.

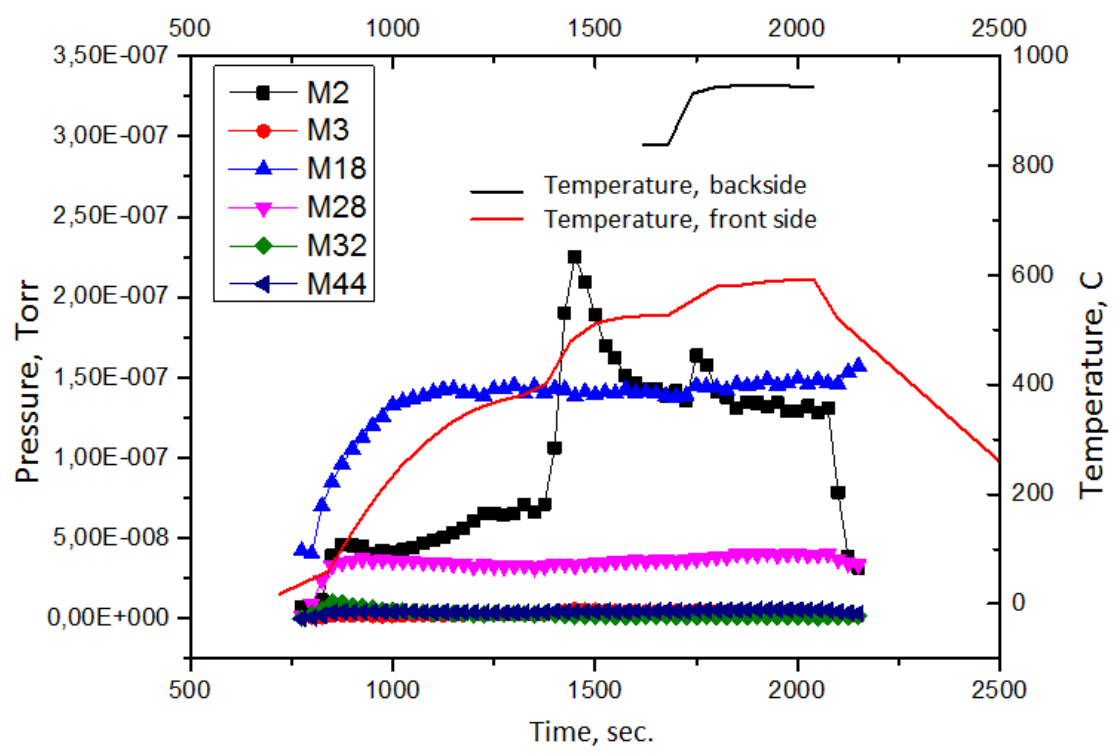

Figure 6 - Depending of gas phase composition change in interaction chamber under stage heating of vanadium sample

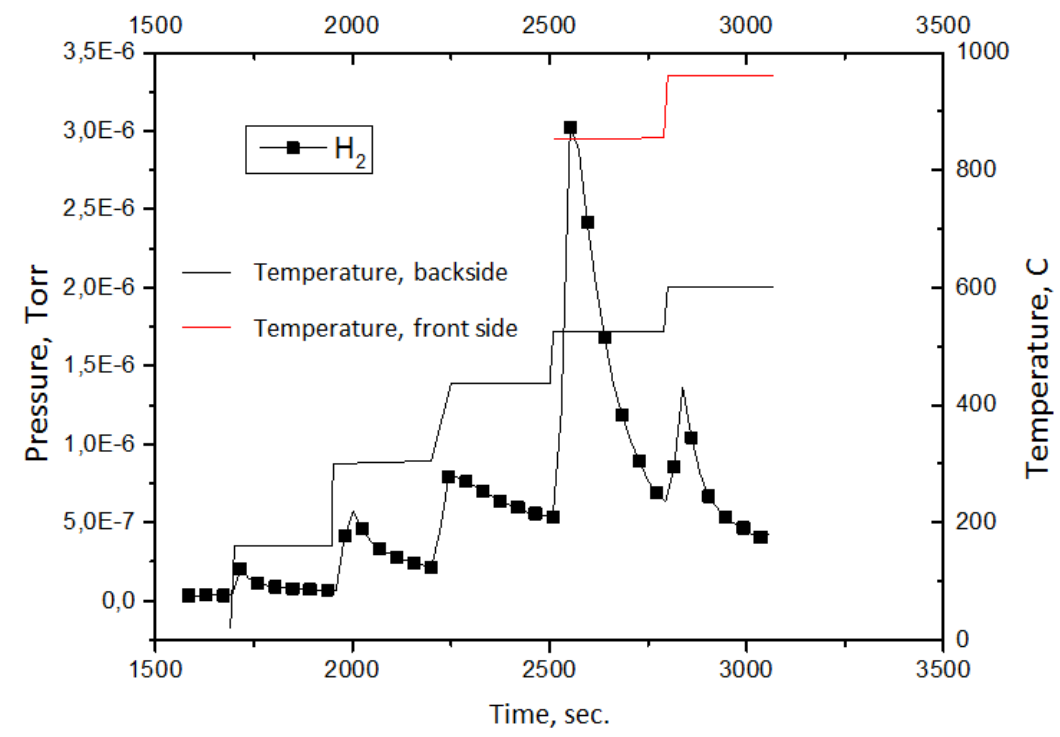

Figure 7 - Change depending of hydrogen partial pressure in interaction chamber on vanadium sample stage heating 


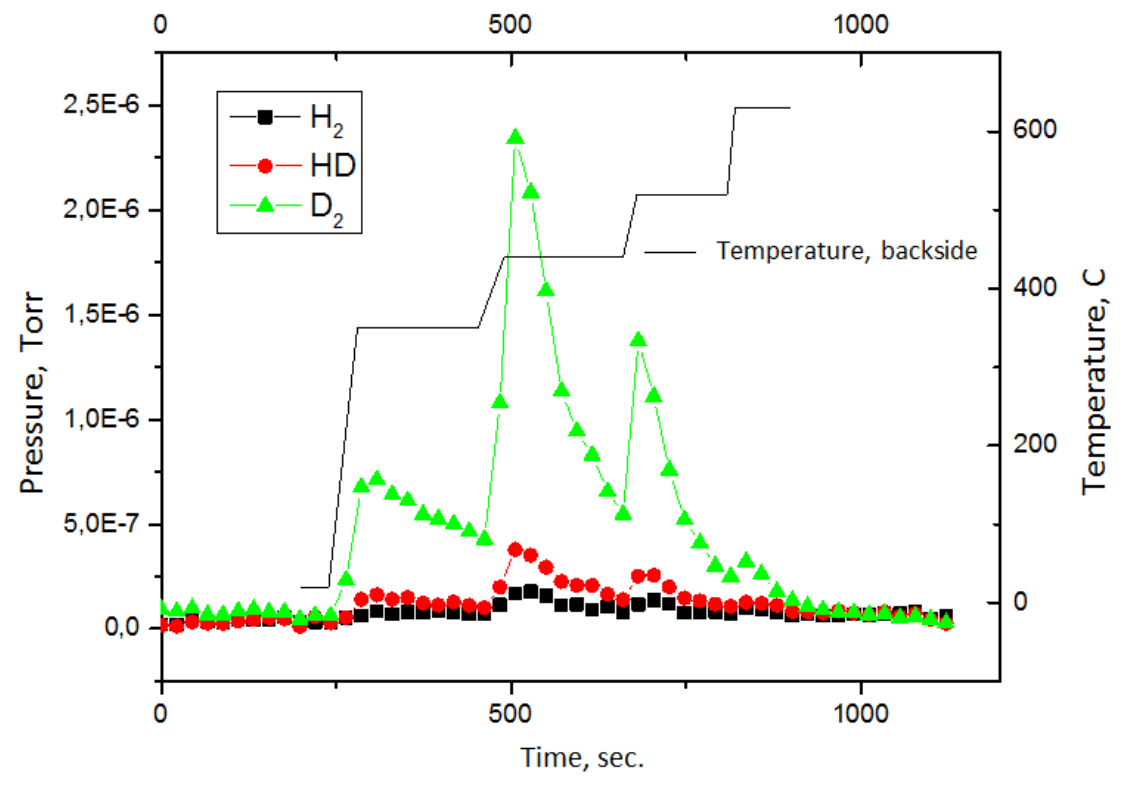

Figure 8-Gas release depending of hydrogen isotopes on stage heating of preliminary saturated vanadium sample

\section{Results and discussion}

Gas release depending analysis on stage heating. Analysis of deuterium gas release depending from vanadium sample was conducted within the assumption of release diffusion mode predominance and based on known approaches to solution of onedimensional diffusion equation.

It is known that, Fick's equations are main equations described hydrogen mass transfer in metals. They are written as follows for onedimensional case:

$$
\begin{aligned}
& J(x, t)=-D(t) \frac{\partial C(x, t)}{\partial x} \\
& \frac{\partial C(x, t)}{\partial t}=D(t) \frac{\partial^{2} C(x, t)}{\partial x^{2}}
\end{aligned}
$$

First equation describes gas penetration rate through environment surface unit. Second equation determines hydrogen accumulation at certain point of environment as function of time $t$.

Depending on conditions at interface gas is solid $x=0, x=1$, equation (1) is Cauchy problem with different boundary conditions.
First order boundary conditions. In this case is generally believed that the concentration at boundary layers is equal to equilibrium solubility. Then for diatomic gas:

$$
\begin{aligned}
& \left.C_{0}\right|_{t \geq 0}=\Gamma \sqrt{P_{0}}, x=0 \\
& \left.C_{l}\right|_{t \geq 0}=\Gamma \sqrt{P_{\partial}}, x=l
\end{aligned},
$$

where $\Gamma=\Gamma_{0} \exp \left(-H_{S} / R T\right)$ - solubility, referred to pressure unit, $P_{0}$ and $P_{l}$ - hydrogen pressure on boundaries (on membrane inlet and outlet sides, as an example). Physically, first order boundary conditions mean that interphase process rate is extremely great in comparison with diffusion mass transfer rate.

Second order boundary conditions are set through diffusant flow at membrane inlet and outlet, equal to constant value:

$$
\begin{aligned}
& -D \frac{d C}{d x}=J_{0}, x=0 \\
& -D \frac{d C}{d x}=J_{l}, x=l
\end{aligned}
$$


On third order boundary conditions, hydrogen concentration at membrane boundary layers is set as flow function through membrane and pressure in gaseous phase. Third order boundary conditions are divided into linear and non-linear relate to hydrogen concentration. Linear transient-free boundary conditions allow to analytically solving transient diffusion problem.

$$
\begin{aligned}
& K_{H} P_{0}-K_{D} C_{0}(t)=J_{0}(t), x=0 \\
& -K_{H} P_{l}+K_{D} C_{l}(t)=J_{l}(t), x=l
\end{aligned}
$$

where $K_{H}$ and $K_{D^{-}}$generalized constants of saturation and degasification relatively. For more correct process description, due to associative character of desorption, non-linear transient third order boundary conditions should be used, considering degasification rate as proportional to $C^{2}$. Then boundary conditions could be written as equation system, describing flow balance on membrane surface and boundary layers:

$$
\begin{gathered}
s \boldsymbol{\mu} p-b N_{0}^{2}-\rho N_{O}+\eta C_{0}=0, x=0 \\
\eta C_{l}-\rho N_{l}-b N_{l}^{2}=0, x=l
\end{gathered}
$$

where $\rho$ - constant of hydrogen transfer from adsorbed to absorbed state, $\eta$ - constant of hydrogen atom outlet rate from volume to surface, $s$ - hydrogen adhesion coefficient, equal to the ratio of the adsorbed particle number to the total number of falling particles on gaseous phase surface, $\mu$ - gaskinetic coefficient, equal to molecule numbers, falling on surface unit in time unit on single hydrogen pressure, $b$ - constant of desorption rate, $N_{o}$ and $N_{l}-$ adsorbed atom concentration at membrane inlet and outlet surfaces.

There is not analytical solution of transient-free diffusion problem on third order boundary conditions.

It is generally assumed that, gas release occurred by diffusion or by first and second order desorption. Gas release process at diffusion mode is described by Fick's equations at classical diffusions or by diffusion equations in defective environment.

For estimation and comparison of obtained kinetics of hydrogen release there can be assumed that deuterium release in above mentioned experiment is determined by diffusion processes (even if it is not such, anyway obtained diffusion coefficient has diffusion effective coefficient meaning and can be characterize kinetic process).

Under boundary conditions $C(0, t)=$ $C_{1}, C(H, t)=C_{2}$ and initial concentration distribution $C(x, 0)$ general solution of diffusion equation in the region of finite sizes is given by the equation:

$$
\begin{aligned}
C(x, t)=C_{1} & +\left(C_{2}-C_{1}\right) \frac{x}{H}+\frac{2}{\pi} \sum_{n=1}^{\infty} \frac{C_{2} \cos n \pi-C_{1}}{n} \sin \frac{n \pi x}{H} \exp \left(-\frac{n^{2} \pi^{2} D t}{H^{2}}\right)+ \\
& +\frac{2}{H} \sum_{m=0}^{\infty} \sin \frac{n \pi x}{H} \exp \left(-\frac{n^{2} \pi^{2} D t}{H^{2}}\right) \int_{0}^{H} C(\xi, 0) \sin \frac{m \pi}{H} \xi d \xi
\end{aligned}
$$

For considered at our experiment desorption case, under continuous pumping conditions it replied to the following boundary and initial conditions:
$C(x, 0)=f(x), \quad C(0, t)=0, \quad C(H, t)=0 . \quad$ Then equation on hydrogen concentration account will be following:

$$
C(x, t)=\frac{2}{H} \sum_{m=0}^{\infty} e^{-\frac{\mu_{m}^{2} D t}{H^{2}}} \int_{0}^{H} C(\xi, 0) \sin \frac{m \pi}{H} \xi d \xi
$$

where $\mu_{m}=m \pi$.

If assume that hydrogen is equal distributed on sample, then there could be another simplification by setting initial condition as: $C(x, 0)=C_{0}$. Then concentration profile will be determined as follows: 


$$
C(x, t)=\frac{4}{\pi} C_{0} \sum_{k=0}^{\infty} \frac{1}{2 k+1} e^{-\frac{(2 k+1)^{2} \pi^{2} D t}{H^{2}}} \cdot \sin \frac{(2 k+1) \pi}{H} x
$$

Considering that diffusion coefficient (see Figure 9) has Arrhenius temperature depending there can be conducted the variation of pre-exponential factors and diffusion activation energy for modeling of obtained gas release depending by recurring:

$$
D=D_{0} \exp \left(-\frac{E_{d}}{R T}\right)
$$

Here $D_{0}$ - pre-exponential factor of diffusion coefficient, $E_{d}$ - diffusion activation energy, $R-$ universal gas constant, $T$ - temperature.

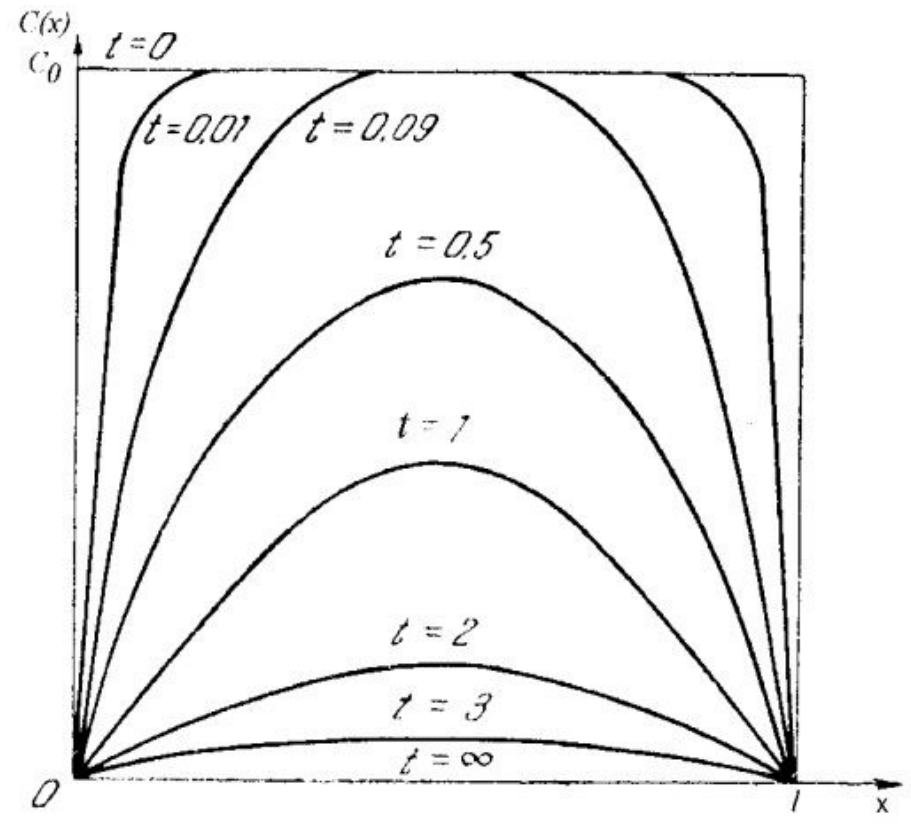

Figure 9 - Diffusion from finite bodies with joint borders (desorption from plates)

It should be noted separately, that 2 decay curves of diffusant flow from samples obtained at a different temperature is necessary for modeling. Then first peak computing will be for temperature values $\mathrm{T} 1$ by equation (8), and for second peak of temperature values $\mathrm{T} 2$ by equation (7) where initial condition will be defined as $C(x, 0)=C(x, t 1) ; \mathrm{t} 1$ - changing time of sample temperatures from $\mathrm{T} 1$ to $\mathrm{T} 2$.

This computation and variation was conducted for experiment on deuterium release from vanadium sample. (Modeling data of deuterium flow decay on sample temperature of $500{ }^{\circ} \mathrm{C}$ is given in Figure 10).

Obtained Arrhenius depending of deuterium diffusion of effective coefficient: $\mathrm{D}(\mathrm{T})=(5 \pm 1)^{*}$ $10^{-8 *} \exp ((12530 \pm 1000) /(\mathrm{R} * \mathrm{~T}))$ coincide on volume order with number of literature sources [15-21].
However we believe that, it is more appropriate for distribution modeling of hydrogen isotope concentration on sample at all experiment stages on saturation/release, to conduct by modern software platforms. COMSOL Multiphysics environment was considered at level of estimation possibility of such modeling.

Potentially, it allows to join two tasks: diffusion and thermal balance in a three-dimensional body (at researched sample) and get temporary distribution depending of sample temperature and hydrogen concentration in sample.

At the present stage of development of experiment analysis means on saturation and gas release, in the basis of COMSOL Multiphysics software environment, thermophysical computations 
of temperature field distribution on sample during impacting electron beam on its surface.

Transient heat transfer model was used during computation of considering case of radial heat:

$$
\rho C \frac{\partial T}{\partial t}+\nabla q=\mathrm{Q} ; \quad q=-k \nabla T
$$

where $\rho$ - thermal conductivity material density (environment); $C$ - material thermal capacitance; $T$ temperature; $q$ - heat flow; $k$ - thermal conductivity coefficient; Q - heat source; $\nabla$ - divergence operator(vector).

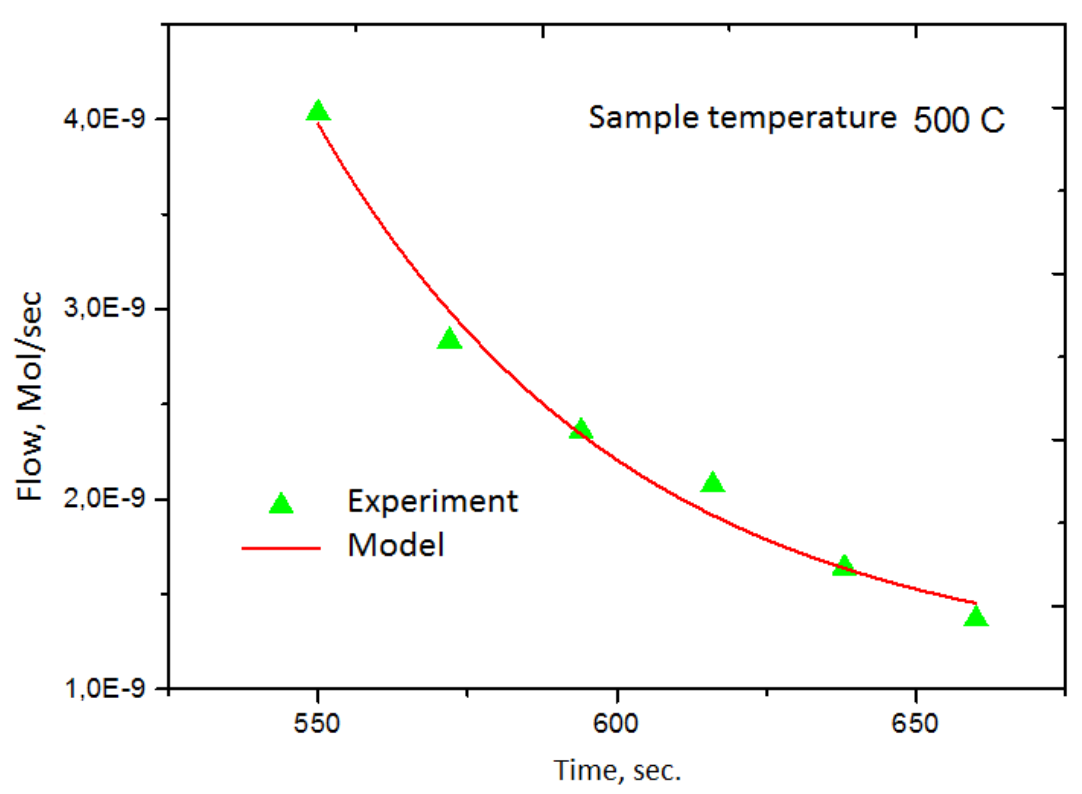

Figure 10 - Results of modeling deuterium release from vanadium sample at a sample temperature of $500{ }^{\circ} \mathrm{C}$

Design model geometry was given in Figure $11 \mathrm{~A}$. Disc type sample from $10 \mathrm{~mm}$ diameter and $2 \mathrm{~mm}$ thickness vanadium was given in the form of half section on axis Z. Sample heating was conducted by supplying thermal energy to sample upper surface: all surface of sample was heated. Outer side of disc circle is cooled by water at the temperature of $50^{\circ} \mathrm{C}$. Outer and lower surface of discs radiate thermal energy by the law $q=$ $-\varepsilon \sigma\left(T_{o}^{4}-T^{4}\right)$, where $\varepsilon-$ material emissivity; $\sigma$ - StefanBoltzmann constant, $\mathrm{T}_{0}-$ environment temperature (installation), is equal to $50^{\circ} \mathrm{C}$.
Further power of sample radial heat was set for computation in $\mathrm{W} / \mathrm{mm}^{2}$.

Three-dimensional space grid sample on the basis of two-dimensional axially symmetric geometry was built for numerical modeling, example of $4 \mathrm{~B}$ SCHEME» which is given in Figure $11 \mathrm{~B}$.

Computation results of temperature field on sample were given in Figures 12, 13. It is shown that generally it reflects experimentally observing effect on sample thickness significant temperature gradient. 


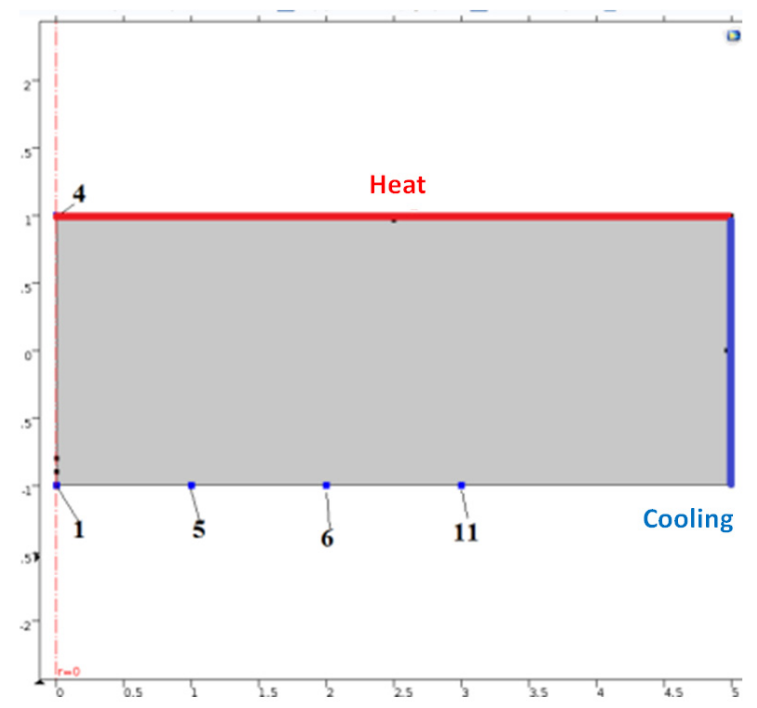

A)

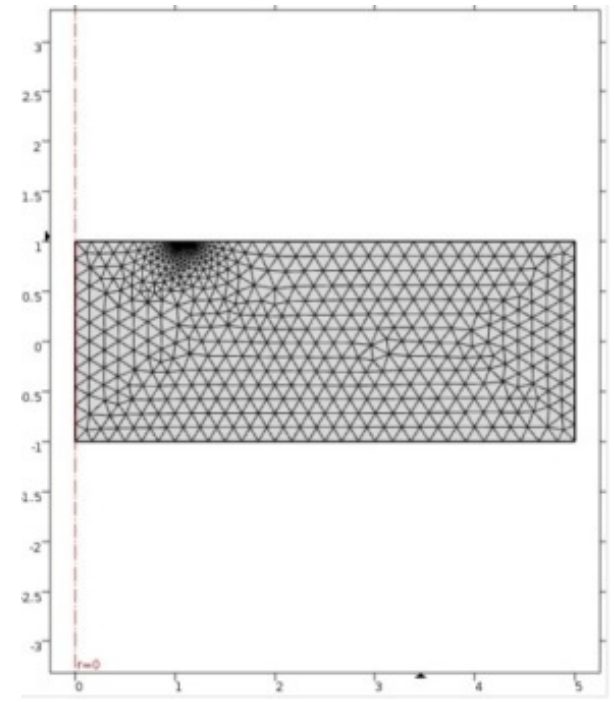

B)

Figure 11 - Sample geometry and calculation model

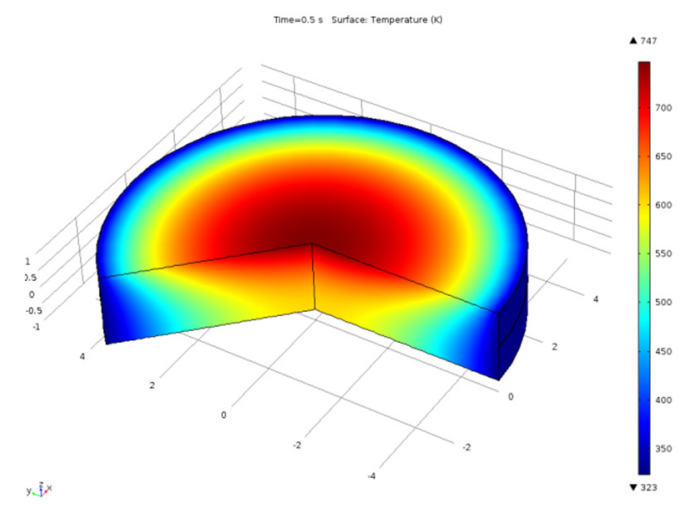

Figure 12 - Temperature field on vanadium sample after $5 \mathrm{sec}$ heating. Beam power is $5 \mathrm{~W} / \mathrm{mm}^{2}$

\section{Conclusions}

Main conclusions on conducted experiments will be following:

1) Possibility of conduction gas release experiments of preliminary irradiated samples on plasma-beam installation was approved and demonstrated; main procedures of such test conduction were determined; methods to analysis and development of saturation/gas release experiment results were introduced.

2) Absorbed deuterium atom quantity at ion radiation time and effective coefficient of deuterium

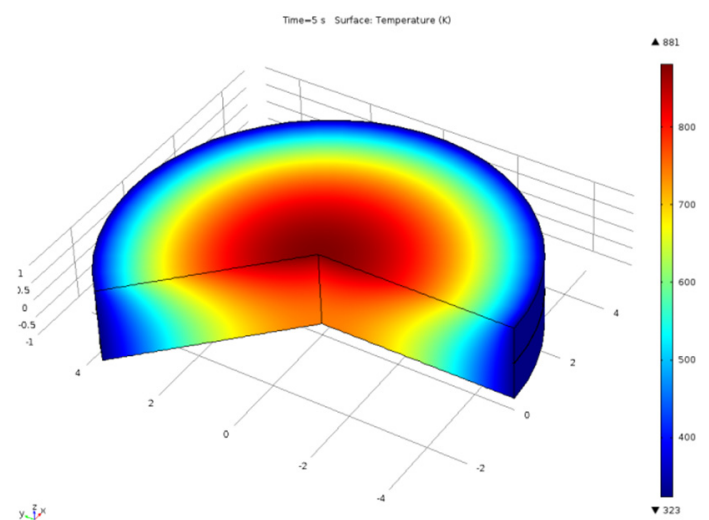

Figure 13 - Temperature field on vanadium sample after $5 \mathrm{sec}$ heating. Beam power is $5 \mathrm{~W} / \mathrm{mm}^{2}$

diffusion in vanadium were detected according data on methodical saturation/gas release experiments with vanadium sample.

3) Technical requirements on gas release experimental conditions, and to its procedure conduction as follows:

- hydrogen isotope quantity in researched saturated sample should be less than $3 * 10^{-10} \mathrm{~mol}$;

- sample should be more than $2 \mathrm{~mm}$ thick;

- sample should be annealed up to temperature of $100{ }^{\circ} \mathrm{C}$ more than assumed temperature at gas release experiments, before saturation experiment conduction 
- registration of temperature on sample front and reverse side within the range of $500-1000{ }^{\circ} \mathrm{C}$ is necessary during experiment conduction.

- gas release experiments are necessary to conduct at no less than 2 different temperatures (preferable at temperatures of $500{ }^{\circ} \mathrm{C}$ and $1000^{\circ} \mathrm{C}$ )

- time between sample saturation and its degasification should be no less than 10 minutes for decreasing of background gas pressure in interaction chamber;

- sample warm-up by electron beam is necessary to conduct under beam defocusing conditions at all sample surface

- mass-spectrum registration should be conducted at turning on SEM within the range of mass number 2 up to mass number 28, with rate of not less than 1 mass-spectrum in 25 seconds.

4) Important tasks were formulated, solution of which could significantly improve quality of integral material testing researches on plasma-beam installation. That important tasks are as follows:
- Conduction of the experiments on calibration of mass-spectrometer system of plasma-beam installation for different gas and different installation pumping configuration;

- Development and test conduction on defining ion beam parameters;

- Making a system of sample line heating by electron beam, for the purpose of realization of tests on sample thermal desorption in line heating mode;

- Development and making method of analysis and modeling experiment results on sample saturation/degasification on plasma-beam installation with COMSOL Multiphysics software platform.

\section{Acknowledgements}

The work was carried out within the framework of the grant \# AP05133148 of the Ministry of Education and Science of the Republic of Kazakhstan.

\section{References}

1. A. Loarte, G. Saibene, R. Sartori et al. Transient heat loads in current fusion experiments, extrapolation to ITER and consequences for its operation // J. Physica Scripta. - 2007. -Vol. 128. -P. 222 - 228.

2 C.H. Skinner, A.A. Haasz, V.Kh. Alimov et al. Recent advances on hydrogen retention in ITER's plasma-facing materials: beryllium, carbon, and tungsten // J. Fusion Sci. Technol. - 2008. -Vol. 54. - P. 891-945.

3 R.A. Pitts, S. Carpentiera, F. Escourbiaca et al. Physics basis and design of the ITER plasma-facing components // J. of Nuclear Materials. - 2011. - Vol. 415. - P. 957-964.

4 A.A. Mazayev, R.G. Avarbe, Y.N. Vilk et al. Solubility of hydrogen in tungsten at high temperature and pressures // Izv. Akad.Nauk.USSR Metally. - 1968. - Vol. 6. - P. 233-238.

5 R. Fraunfelder. Permeation of hydrogen through tungsten and molybdenum // J. Chem. Phys. - 1967. - Vol. 48. P. 3955-3965.

6 G. Benamati, E. Serra, and C.H. Wu. Hydrogen and deuterium transport and inventory parameters through W and W-alloys for fusion reactor applications // J. Nucl. Mater. - 2000. - Vol. 283-287. - P. 1033-1037.

7 A.P. Zakarov, V.M. Sharapov, E.I. Evko. Hydrogen permeability of polycrystalline and monocrystalline molybdenum and tungsten // Soviet Mater. Sci. - 1973. - Vol. 9. - P. 149-153.

8 D.T. Peterson, H.M. Herro. Hydrogen and deuterium diffusion in vanadium-titanium alloys // Metallurgica Transactions A. - 1987. - Vol. 18A. - P. 249-254

9 Anderl R.A., Longhurst G.R., Struttmann D.A. Permeation of deuterium, implanted into V-15Cr-5Ti // Journal of nuclear materials. - 1987. - Vol.145. - P. 344-347.

10 Kofstad P., Wallace W.E. Vapor pressure studies of the vanadium-hydrogen system and thermodynamics of formation of vanadium - hydrogen solid solutions // J.Am.Chem.soc. - 1959. - Vol. 81 . - P. 5019-5022.

11 Eguchi T., Morozumi S. Influence of alloying elements on the solubility of hydrogen in vanadium // J. of the Japan Institute of Metals. - 1974. - Vol. 38, №11. - P. 1025-1030.

12 Boes N., Zuchner H. Measurements of the solubility of hydrogen in vanadium, niobium and tantalum with a new electrochemical method // Berichte der Bunsen-Gesellechaft. - 1976. - Vol. 80, № 1. - P. 22-27.

13 Lynch J.F., Reilly J.J., Millot F. The absorption of hydrogen by binary vanadium- chromium alloys // J. Phys. Chem. Solids. - 1978. - Vol. 39. - P. 883-890.

14 Bleichert H., Wenzel H. Variable solubility of the hydrogen isotopes, protium, deuterium, tritium in vanadium // Phys. Stat. Sol. - 1987. - Vol 144, № 1. - P. 361-373. 
15 Reiter F., Forcey K.S., Gerwasini A. A compilation of tritium - material Interactions parameters in fusion reactor materials // Commission of the European Communities, ISPRA. Joint research center report EUR 15217 EN. - 1993. $54 \mathrm{p}$.

17 Schaumann G., _lkl J.V., Alefeld G. The diffusion coefficients of hydrogen and deuterium in vanadium, niobium and tantalum by Gorsky-effect measurements // Phys. Stat. - 1970. - Vol. 42. - P. 401-408.

19 Freudenberg U., lkl J. V., Bressers J., Alefeld G. Influence of impurities on the diffusion coefficient of hydrogen and deuterium in vanadium // Scripta Metallurgica. - 1978. - Vol.12. - P. 165-167.

20 Tanaka S., Kimura H. Solubility and diffusion of hydrogen in vanadium and its alloys around room temperature // Trans. JIM. - 1978. - Vol. 20. - P. 647-652.

21 Pine D.J., Cotts R.M. Diffusion and electrotransport of hydrogen and deuterium in vanadium-titanium and vanadium-chromium alloys // Physical review B. - 1983. - Vol. 28. - № 2. - P. 641. 